\title{
Supplement to The tropical route of QBO teleconnections in a climate model WCD
}

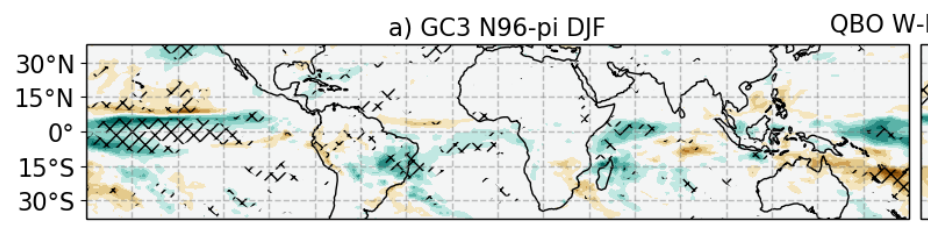

c) GC3 N96-pi MAM

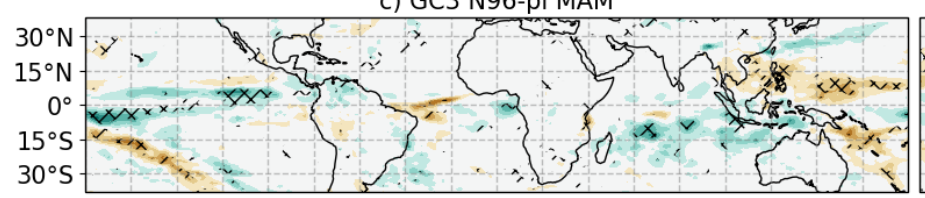

e) GC3 N96-pi JJA

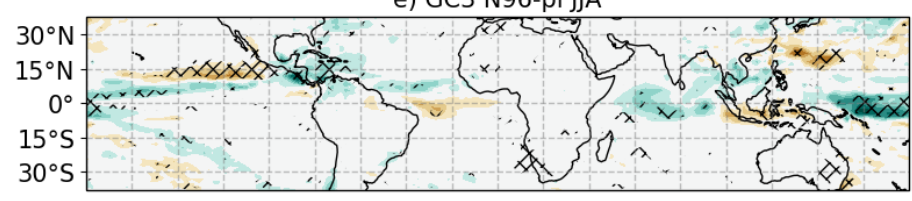

g) GC3 N96-pi SON

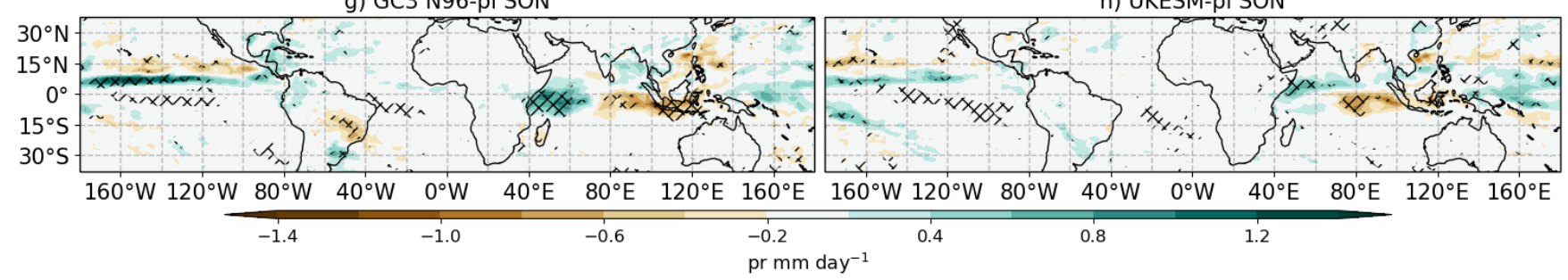

Figure S1. Seasonal mean precipitation differences between QBO W-E phases in (left) GC3 N96-pi and (right) UKESM-pi for (a, b) DJF, (c, d) MAM, (e,f) JJA and (g, h) SON. Hatching denotes statistically significant differences to the $95 \%$ confidence level using a bootstrapping with replacement test. b) UKESM-pi DJF

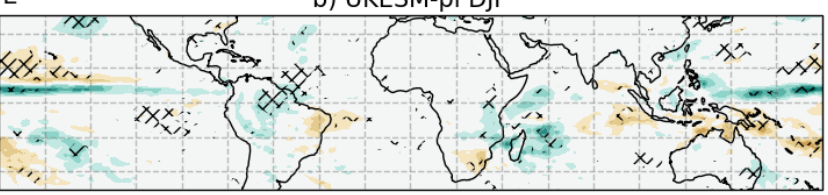

d) UKESM-pi MAM

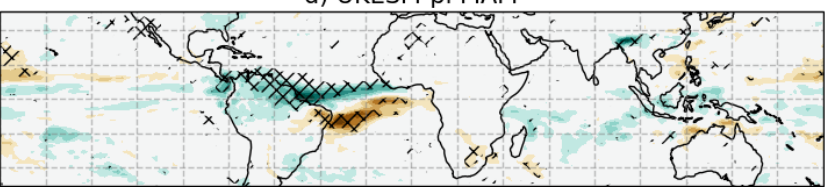

f) UKESM-pi JJA

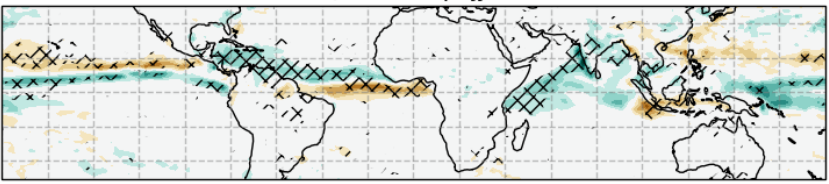

h) UKESM-pi SON 
$\begin{array}{ll}\text { a) GC3 N96-pi DJF } & \text { QBO W-E }\end{array}$

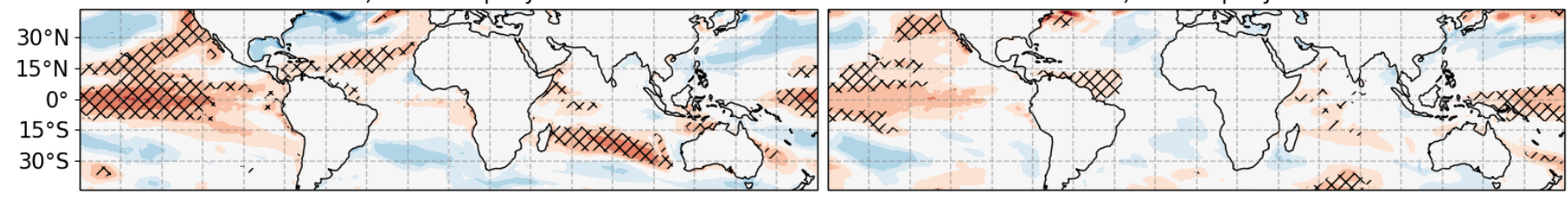

c) GC3 N96-pi MAM

d) UKESM-pi MAM
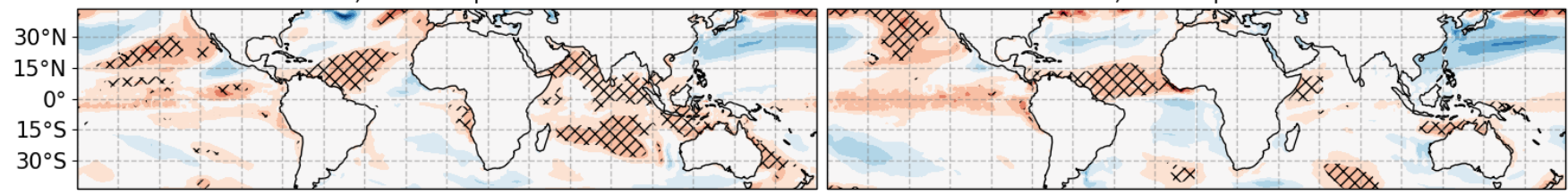

e) GC3 N96-pi JJA

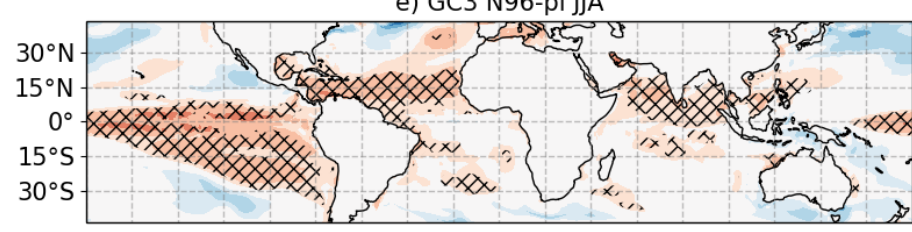

f) UKESM-pi JJA

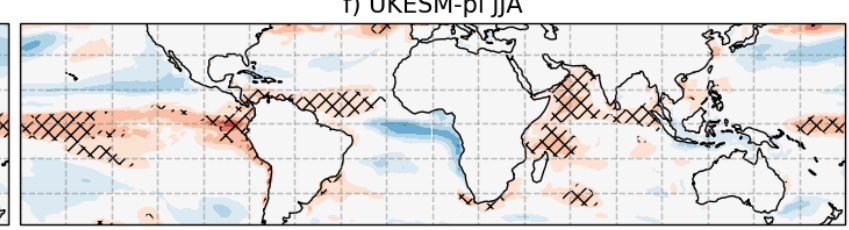

g) GC3 N96-pi SON

h) UKESM-pi SON

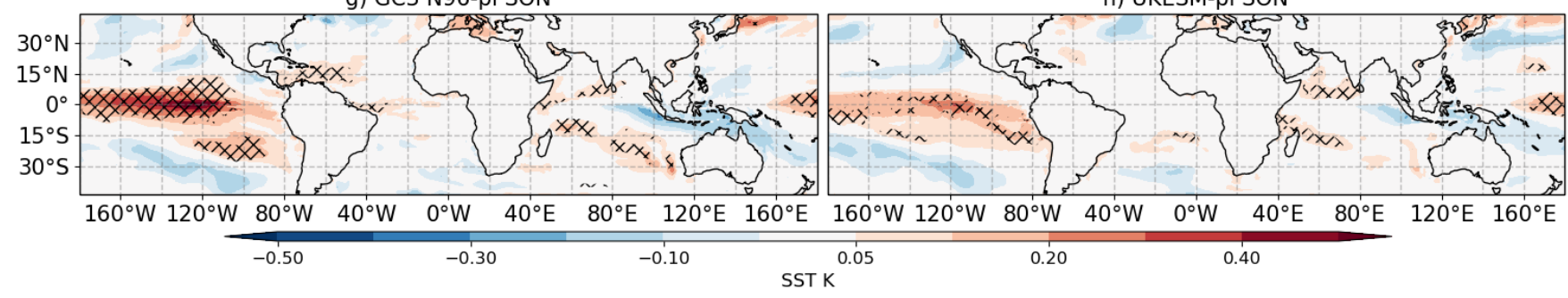

Figure S2. SST composite differences for (left) GC3 N96-pi and (right) UKESM-pi for the seasons of (a-b) DJF, (c-d) MAM, (e-f) JJA and (g-h) SON. 
a) QBO Simple OLS $\beta_{i}$

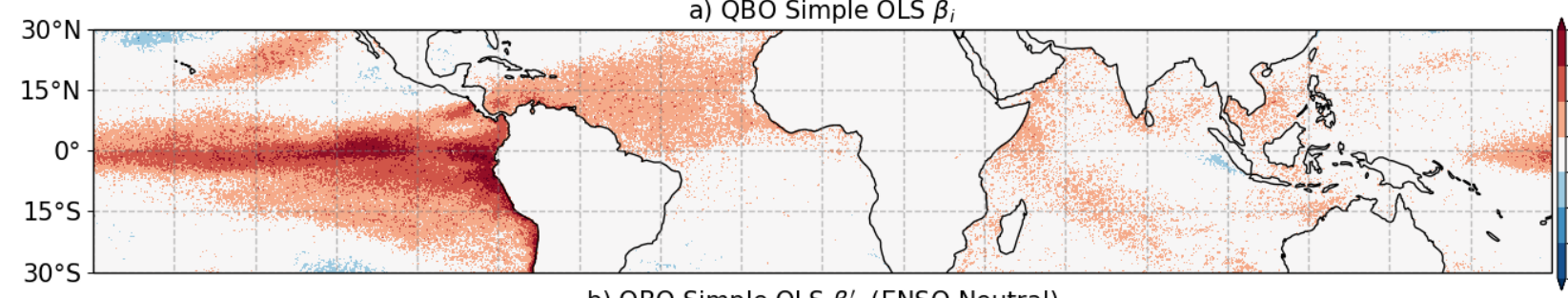

0.20

0.15

b) QBO Simple OLS $\beta_{i}^{\prime}$ (ENSO Neutral)
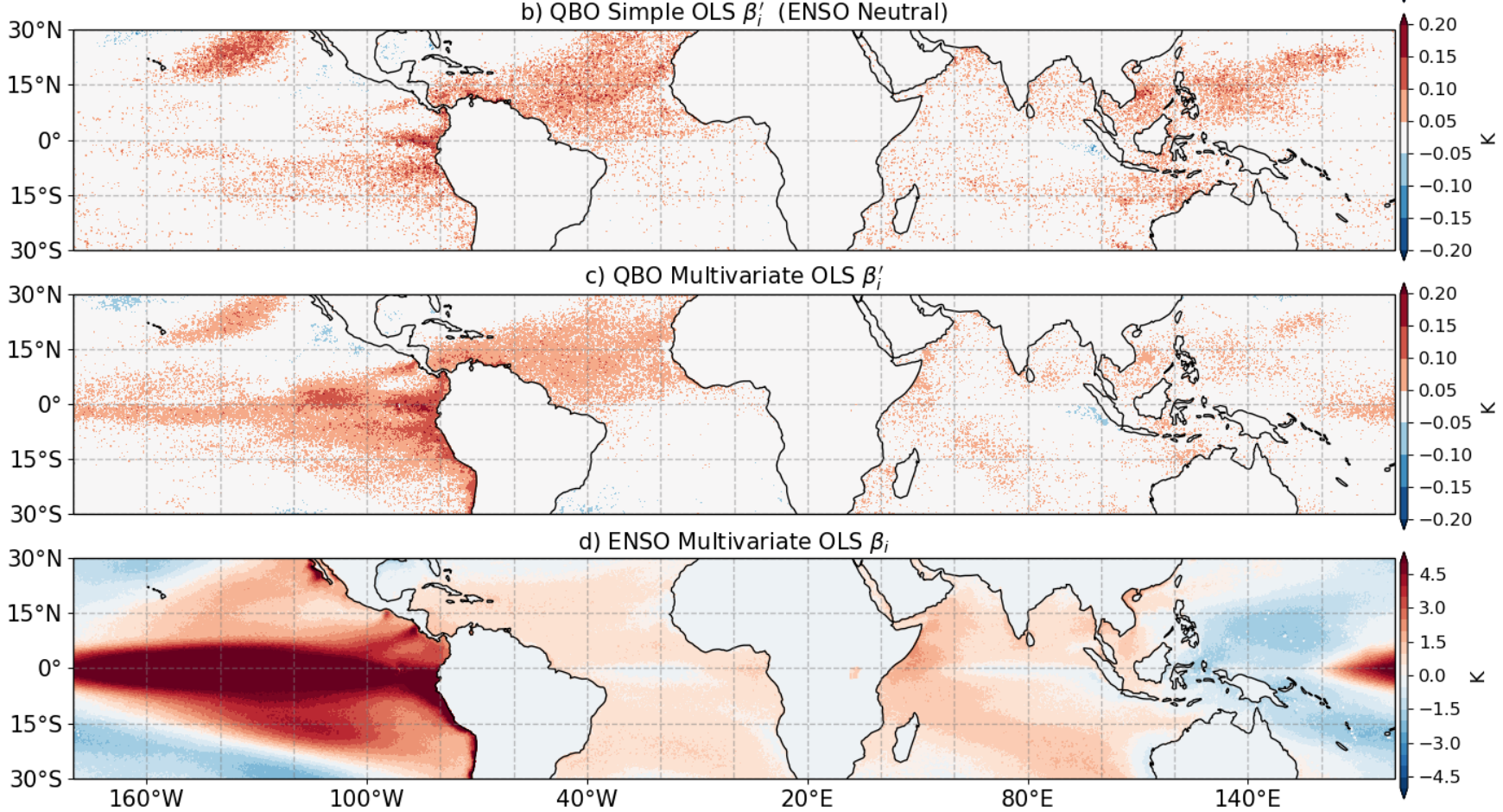

Figure S3. Regression analysis as in Figure 5 of the main manuscript but for sea-surface temperatures (SSTs). 


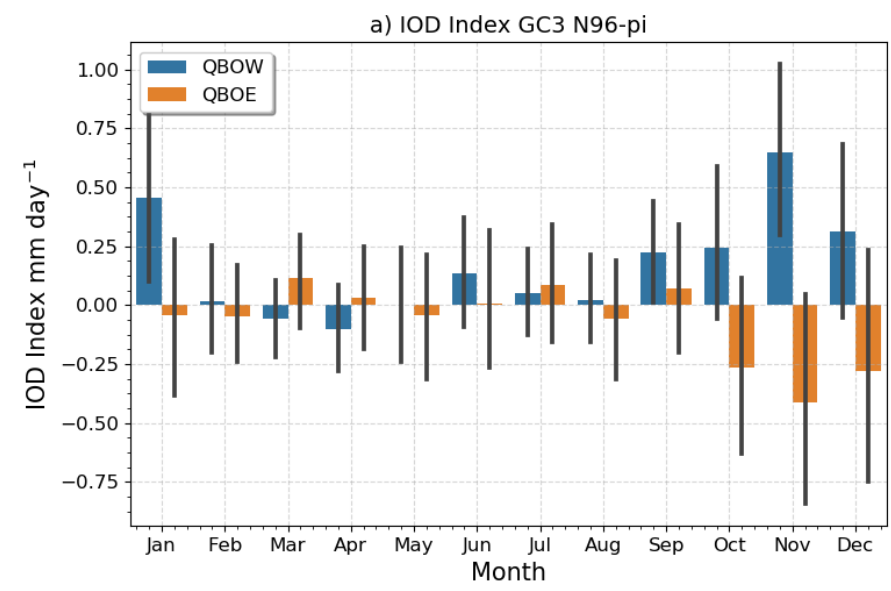

c) UKESM-pi IOD Index

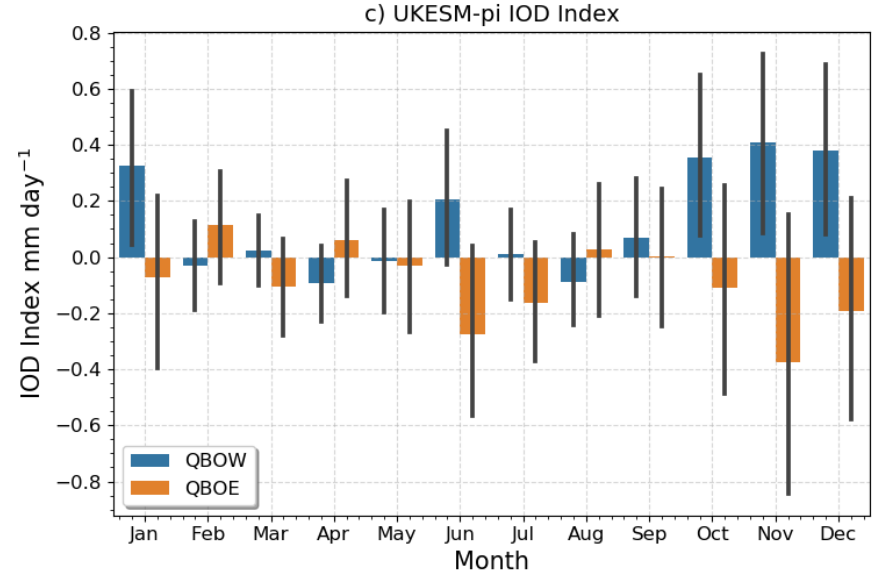

b) ENSO index GC3 N216-pi

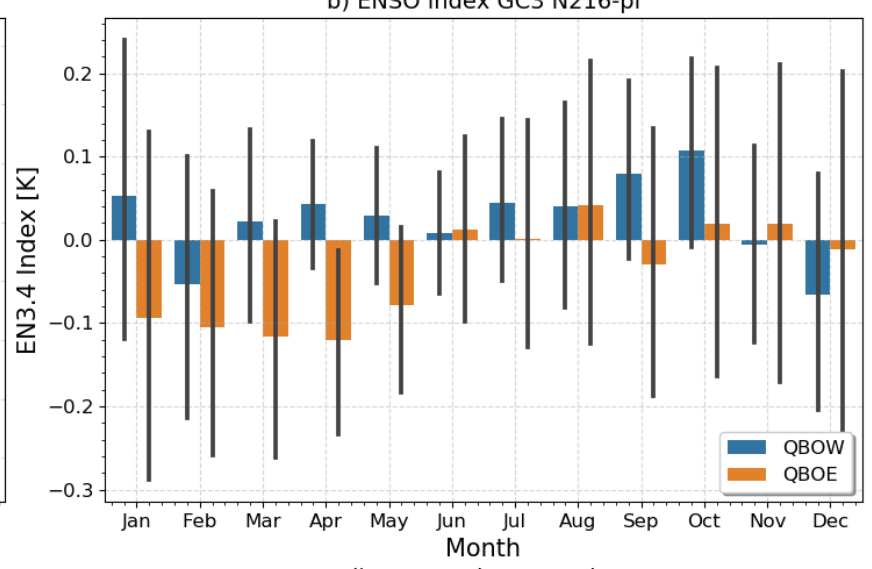

d) UKESM-pi ENSO Index

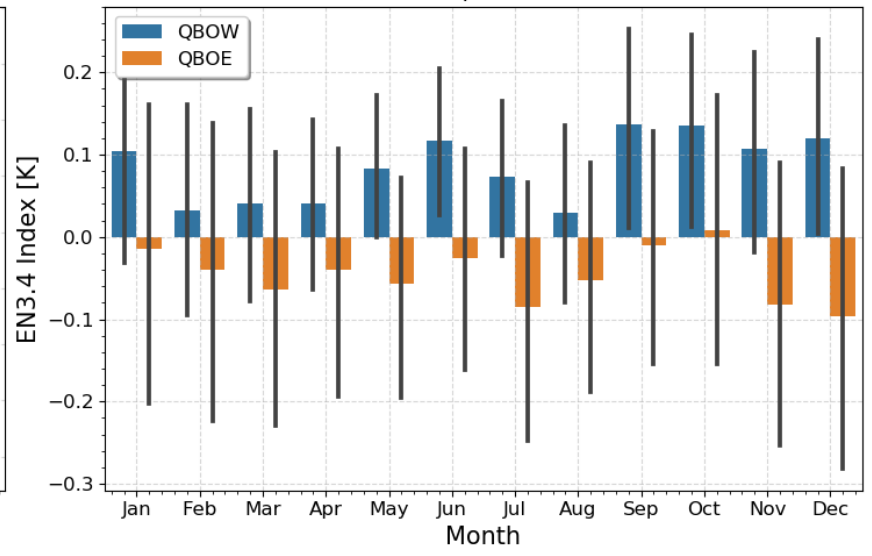

Figure S4. As in Figure 6 of the main manuscript but for (a, b) GC3 N96-pi and (c, d) UKESM-pi. 
a) Atlantic GC3 N216 piControl-ERA5

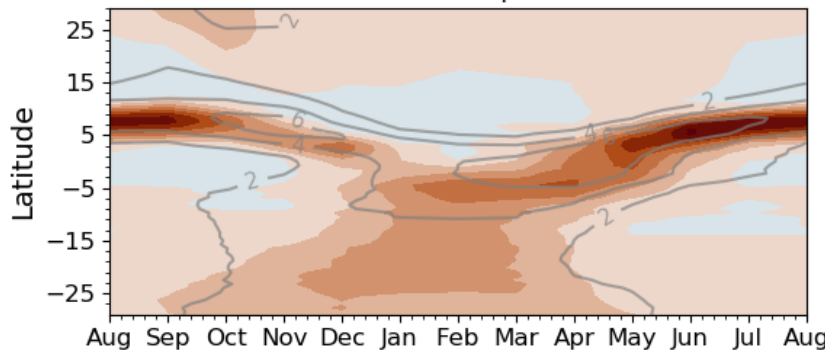

c) Atlantic ERA5 QBO W-E

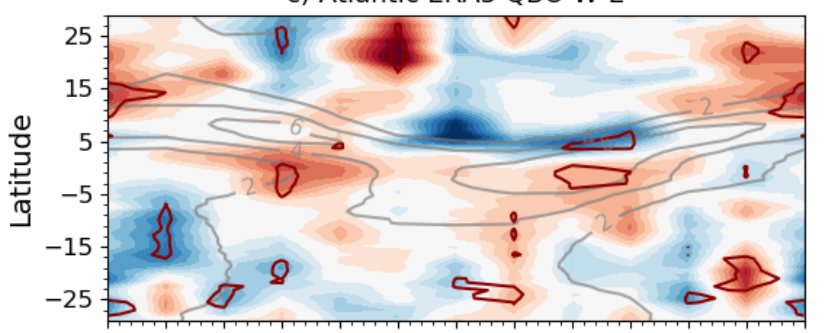

Aug Sep Oct Nov Dec Jan Feb Mar Apr May Jun Jul Aug e) Atlantic GC3 N216 piControl QBO W-E

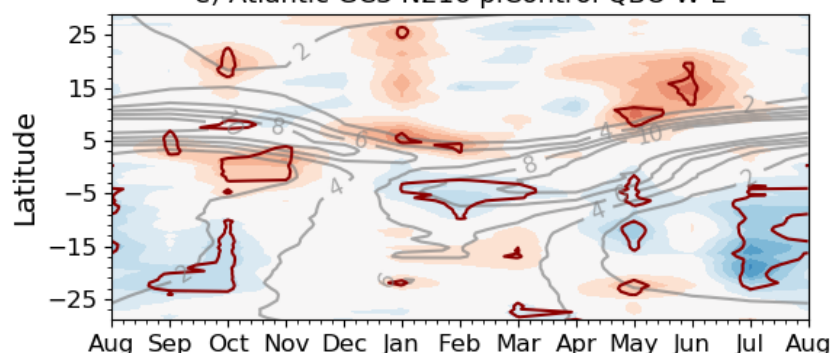

b) Central Pacific GC3 N216 piControl-ERA5

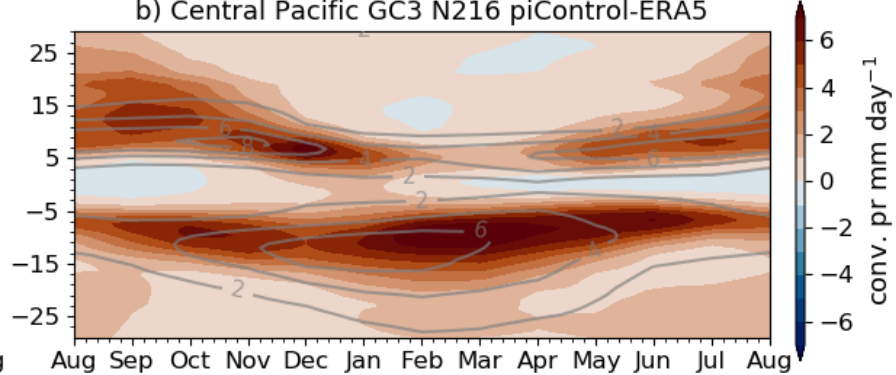

d) Central Pacific ERA5 QBO W-E

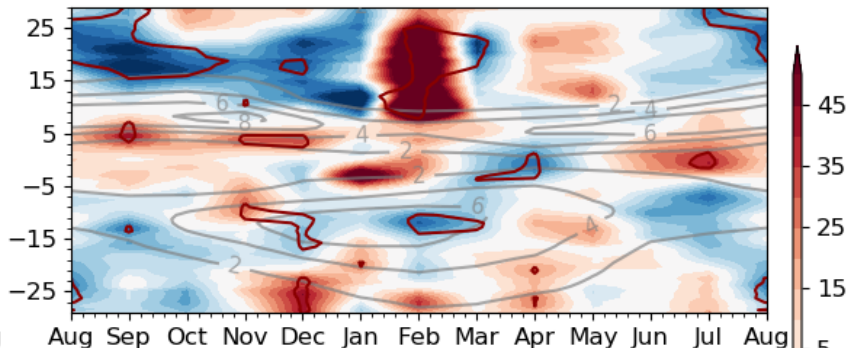

Aug Sep Oct Nov Dec Jan Feb Mar Apr May Jun Jul Aug|- 5 ○

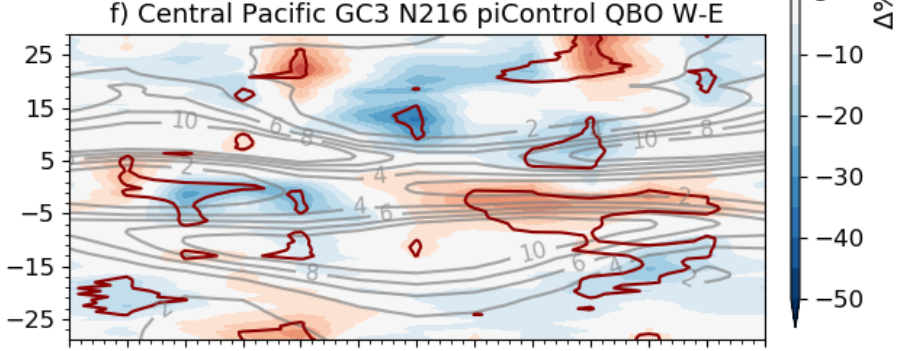

Aug Sep Oct Nov Dec Jan Feb Mar Apr May Jun Jul Aug

Figure S5. As in Figure 9 of the main manuscript but only for Neutral years only. 